\title{
Electromagnetic energy harvester for monitoring wind turbine blades
}

\author{
B. S. Joyce ${ }^{1}$, J. Farmer ${ }^{1}$ and D. J. Inman ${ }^{2}$ \\ ${ }^{1}$ Center for Intelligent Materials Systems and Structures, Virginia Tech, Blacksburg, Virginia, USA \\ ${ }^{2}$ Department of Aerospace Engineering, University of Michigan, Ann Arbor, Michigan, USA
}

\begin{abstract}
The long composite blades on large wind turbines experience tremendous stresses while in operation. There is an interest in implementing structural health monitoring (SHM) systems inside wind turbine blades to alert maintenance teams of damage before serious component failure occurs. This paper proposes using an energy harvesting device inside the blade of a horizontal axis wind turbine to power a SHM system. The harvester is a linear induction energy harvester placed radially along the length of the blade. The rotation of the blade causes a magnet to slide along a tube as the blade axis changes relative to the direction of gravity. The magnet induces a voltage in a coil around the tube, and this voltage powers the SHM system. This paper begins by discussing motivation for this project. Next, a harvester model is developed, which encompasses the mechanics of the magnet, the interaction between the magnet and the coil, and the current in the electrical circuit. A free fall test verifies the electromechanical coupling model, and a rotating test examines the power output of a prototype harvester. Copyright (C) 2013 John Wiley \& Sons, Ltd.
\end{abstract}

\section{KEYWORDS}

electromagnetic; energy harvesting; wind turbine blades; structural health monitoring

Correspondence

B. S. Joyce, Center for Intelligent Materials Systems and Structures, Virginia Tech, 310 Durham Hall, Blacksburg, Virginia 24061, USA.

E-mail: bjoyce1@vt.edu

Received 22 February 2012; Revised 27 November 2012; Accepted 21 January 2013

\section{INTRODUCTION AND LITERATURE REVIEW}

Large-scale wind turbines are designed with long blades to increase the achievable power output of the turbine. These blades undergo years of fatigue, which can lead to crack formation. ${ }^{1}$ Destruction of a wind turbine blade can be disastrous and results in costly downtime. ${ }^{2}$ Therefore, there is a desire to utilize structural health monitoring (SHM) equipment to examine the blades for damage while the wind turbine is in operation. ${ }^{3}$ Batteries inside the blade could supply power to the equipment, but batteries would require regular replacement. An alternative power source would be an energy harvesting device inside the wind turbine blade. The harvester would convert the rotation of the rotor into electrical power useful for the SHM equipment. This approach allows one to design a self-powering SHM and energy harvester system for easier installation inside the blade.

Wind turbines with power outputs between 1 and $5 \mathrm{MW}$ turn at speeds up to $20 \mathrm{rpm} .^{4,5}$ Any useful energy harvester would have to function under these extremely low driving frequencies. Several researchers have examined harvesters for rotating environments. In 2009, a research group from Los Alamos National Laboratory examined several energy harvesting techniques to power a SHM device inside a wind turbine blade. ${ }^{6}$ Although electromagnetic energy harvesting was not studied, piezoelectric harvesters were used to capture energy from the spinning rotor. An L-shaped harvester had a first natural frequency of $2.7 \mathrm{~Hz}(162 \mathrm{rpm})$, far above the driving frequencies expected in a large-scale wind turbine. Conrad ${ }^{7}$ developed an electromagnetic energy harvester based on a slider-augment pendulum. With the device placed $1 \mathrm{~m}$ from the center of rotation, the harvester produced about $80 \mathrm{~mW}$ to a $48 \Omega$ load resistor at $150 \mathrm{rpm}$. Gu and Livermore ${ }^{8}$ and Manla, et al. ${ }^{9}$ have examined placing energy harvesters inside automobile tires. However, these harvesters produced only a few tens of microwatts at operating speeds below $200 \mathrm{rpm}$. 
This paper examines using a linear electromagnetic energy harvester to power the SHM equipment. The rotation of the wind turbine causes a magnet to slide along the length of a tube. The motion of the magnet induces a voltage in coil around the tube. This voltage energizes an electrical circuit and powers the SHM system. This energy harvesting device is capable of operating at the extremely low rotation speeds of large-scale wind turbines.

\section{MODEL DEVELOPMENT}

\subsection{Harvester layout and mechanics}

Figure 1(a) shows a schematic of the energy harvester assembly, and Figure 1(b) shows the harvester inside a wind turbine blade. The design consists of a magnet inside a tube that is mounted radially along the length of the turbine blade. As the rotor spins, the changing orientation of the blade causes the magnet to slide along the tube. Figure 1(c) shows a free body diagram of the magnet. Four forces act on the magnet: the force of gravity $(M g)$, an electromagnetic drag force $\left(F_{\mathrm{em}}\right)$, a Coulomb friction force $\left(F_{\mathrm{f}}\right)$, and a normal force $(N)$ from the tube walls. The electromagnetic drag force is produced by the induced magnetic field of the coil interacting with the magnetic field of the magnet.

From Newton's second law of motion in a rotating coordinate frame, the equation of motion governing the magnet is

$$
M \ddot{r}-M \Omega^{2} r+F_{f}+F_{\mathrm{em}}=-M g \cos (\theta),
$$

where $r$ is the radial position of the magnet, $\theta$ is the clockwise angle of the harvester with respect to the vertical position, $M$ is the mass of the magnet, $\Omega$ is rotation speed of the wind turbine in radians per second, $g$ is the acceleration due to gravity, and an overdot denotes differentiation with respect to time. Applying Newton's second law in the $\theta$-direction leads to an expression for the normal force $N$,

$$
N=M(g \sin (\theta)-2 \dot{r} \Omega-r \dot{\Omega}) .
$$

This normal force appears in the expression for the Coulomb friction force. We define $F_{p}$ as the applied force on the magnet, which attempts to counter the force of friction, namely

$$
F_{p}=M \Omega^{2} r-F_{\mathrm{em}}-M g \cos (\theta)
$$

Let $\mu_{\mathrm{s}}$ be the static coefficient of friction between the magnet and the tube wall and $\mu_{\mathrm{k}}$ be the kinetic coefficient of friction. If the magnet is at rest (i.e., $\dot{r}=0$ ) and $\left|F_{\mathrm{p}}\right| \leq \mu_{\mathrm{s}}|N|$, then the magnet will remain at the same radial position and will not slide along the tube. If these conditions are not met, the magnet will move, and the friction force becomes

$$
F_{f}=\mu_{k}|N| \operatorname{sgn}(\dot{r}) \text {. }
$$

The electromagnetic drag force is related to the current $(I)$ in the coil by

$$
F_{\mathrm{em}}=\alpha(r) I
$$

where $\alpha(r)$ is the electromechanical coupling factor. This coupling factor is a function of the radial position of the magnet. This factor will be derived in the next section.

Because the magnet cannot pass through the ends of the tube, the motion of the magnet must account for the imposed boundaries from the tube. Let $r_{1}$ be the radial position of the bottom of the tube and $r_{2}$ be the radial position of the top of the
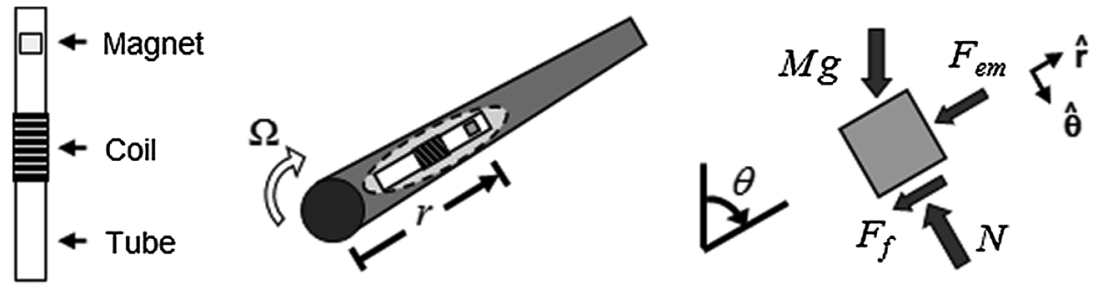

Figure 1. (a) Energy harvester assembly. (b) Energy harvester inside a wind turbine blade. (c) Free body diagram of the magnet. 
tube. If $r$ equals $r_{1}$, then the radial velocity of the magnet cannot be negative (i $\left.\geq 0\right)$. Likewise, if $r$ equals $r_{2}$, then the radial velocity cannot be positive $(\dot{r} \leq 0)$.

\subsection{Electromechanical coupling factor}

Next, the coupling coefficient between the magnet and the coil is derived. The induced voltage $(\varepsilon)$ relates to radial velocity of the magnet $(\dot{r})$ through the following equation:

$$
\varepsilon=\alpha(r) \dot{r}
$$

Often, the coupling factor is approximated as a constant to simplify analysis. ${ }^{10,11}$ However, this is not acceptable for this energy harvester because of the large amplitude of motion for the magnet. By approximating the magnet as a magnetic dipole moment, Faraday's law of induction gives the coupling factor as 12,13

$$
\alpha(r)=\left.\left.\frac{1}{2} \frac{\mu_{0} m l_{w}}{V_{\text {coil }}}\left\{\frac{a}{\sqrt{a^{2}+(r-c)^{2}}}-\ln \left(\sqrt{a^{2}+(r-c)^{2}}+a\right)\right\}\right|_{c=c_{1}} ^{c_{2}}\right|_{a=a_{1}} ^{a_{2}}
$$

where $\mu_{0}$ is the permeability of free space, $m$ is the magnetic dipole moment of the permanent magnet, $l_{\mathrm{w}}$ is the length of wire comprising the coil, $\mathrm{V}_{\text {coil }}$ is the volume of the coil, $c_{1}$ is the radial position of the bottom of the coil, $c_{2}$ is the radial position of the top of the coil, $a_{1}$ is the inner radius of the coil, and $a_{2}$ is the outer radius of the coil.

\subsection{Circuit design}

The coil of the harvester is connected to an electronic circuit. Figure 2 shows a simple load resistor circuit. The coil is treated as a voltage source $(\varepsilon)$ in series with an inductor $\left(L_{\text {coil }}\right)$ and a resistor $\left(R_{\text {coil }}\right)$. The energy produced from the motion of the magnet is dissipated across a load resistor $\left(R_{\text {load }}\right)$. This dissipated energy indicates the potential energy production of the harvester to a SHM system. The simplicity of this circuit is useful for validating the model of the harvester and for studying the harvester's behavior. The simple load resistor circuit is governed by

$$
L_{\text {coil }} \dot{I}+\left(R_{\text {coil }}+R_{\text {load }}\right) I=\varepsilon=\alpha(r) \dot{r}
$$

The power $\left(P_{\text {load }}\right)$ dissipated through the load resistor is

$$
P_{\text {load }}=I^{2} R_{\text {load }}=\frac{V_{\text {load }}^{2}}{R_{\text {load }}},
$$

where $V_{\text {load }}$ is the voltage across the load resistor. The total energy dissipated by the load resistor is the integral of the load power over time.

\subsection{Numerical simulation}

The equations of the harvester model are incorporated into a MATLAB code based on Euler's method for solving differential equations. Figure 3 shows the path of the magnet predicted by the numerical solver for one rotation of an example harvester. The harvester rotates clockwise, and the acceleration due to gravity points down the page. The curves denote the path of the magnet for various rotation speeds, a black dot indicates the magnet's initial location, and the inner and outer rings indicate the tube boundaries. Starting with the tube pointing downward, the magnet sits at the outer tube end. As the harvester rotates,

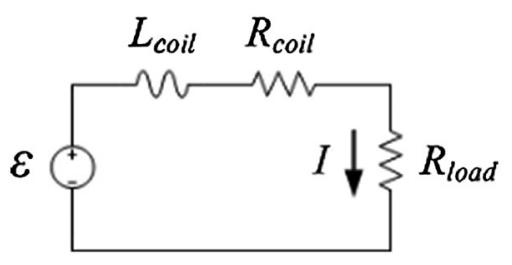

Figure 2. Load resistor circuit. 


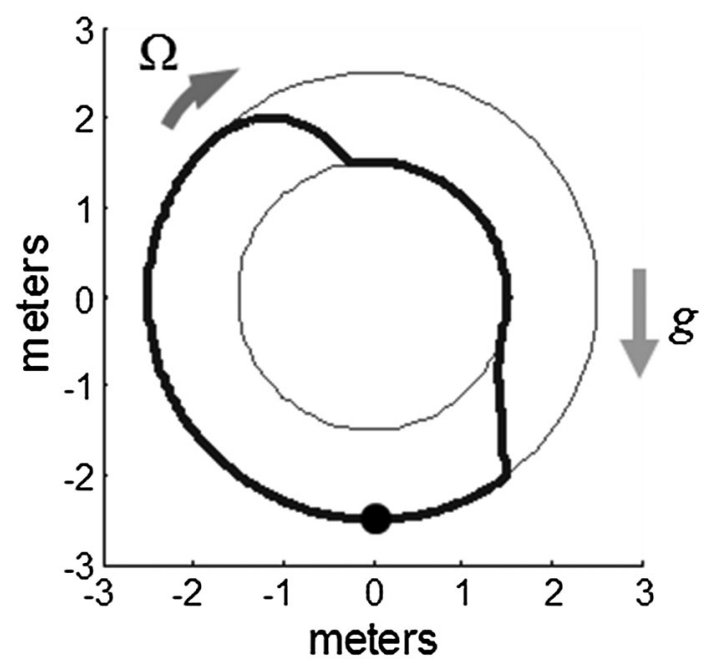

Figure 3. Magnet's path inside the energy harvester for various rotation speeds.

the magnet remains at the outer end until the tube rotates far enough for gravity to overcome the static friction and the centrifugal effects. Then the magnet slides from the outer tube end to the inner end. During this slide, voltages will be induced across any coils along the tube. The magnet will remain at the inner tube end until the tube rotates far enough for gravity to move the magnet again. The magnet slides along the coil twice per rotation for the 20 and $40 \mathrm{rpm}$ cases. For higher speeds, the centrifugal effects on the magnet hinder its motion. The magnet does not fall completely to the bottom of the tube for the $50 \mathrm{rpm}$ case in Figure 3. At $60 \mathrm{rpm}$, the magnet only slightly moves away from the outer tube end. The harvester's power output diminishes in both of these high rotation speed cases.

\section{EXPERIMENTAL VALIDATION}

\subsection{Free fall test}

Before testing the full harvester model, it is necessary to validate the expression for the electromechanical coupling factor. Figure 4(a) shows a free fall test assembly. A cylindrical magnet is dropped vertically through a coil resting on a PVC pipe assembly. The terminals of the coil are connected to a load resistor. This test examines the magnet without the complications of the rotating harvester or friction from the tube wall. Table I lists the parameters for this test. Figure 4(b) shows the total energy dissipated over the entire fall of the magnet using several load resistances. Five trials were performed for each resistance value, and the total energies over the five trials were averaged.

The value of the magnetic dipole moment $m$ was estimated using two methods. First, the dipole moment was approximated through the formula
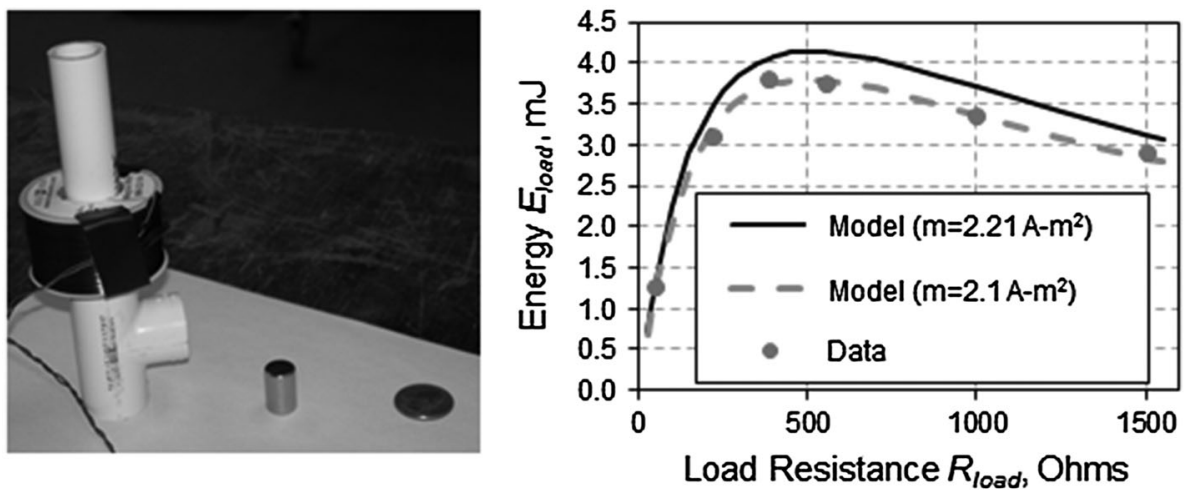

Figure 4. (a) Free fall test assembly and a neodymium magnet. (b) Data and model predictions for the energy dissipated across varying resistances. 
Table I. Parameters for the free fall test. The bottom of the tube is taken to be $r=0$ in.

\begin{tabular}{llcl}
\hline & \multicolumn{1}{c}{ Parameter } & Symbol & Value \\
\hline Magnet & Height & - & $0.75 \mathrm{in}(19.1 \mathrm{~mm})$ \\
& Diameter & - & $0.5 \mathrm{in}(12.7 \mathrm{~mm})$ \\
Coil & Mass & $M$ & $18 \mathrm{~g}$ \\
& Inner radius & $a_{1}$ & $0.509 \mathrm{in}(12.9 \mathrm{~mm})$ \\
& Outer radius & $a_{2}$ & $1.02 \mathrm{in}(28.1 \mathrm{~mm})$ \\
& Coil height & $h$ & $1.17 \mathrm{in}(29.7 \mathrm{~mm})$ \\
& Wire diameter & $D_{\mathrm{w}}$ & $0.0080 \mathrm{in}(0.203 \mathrm{~mm})$ \\
& Coil resistance & $R_{\text {coil }}$ & $426 \Omega$ \\
Tube & Coil inductance & $L_{\text {coil }}$ & $0.998 \mathrm{H}$ \\
Other & Distance from coil to tube bottom & $c_{1}$ & $2.50 \mathrm{in}(63.5 \mathrm{~mm})$ \\
\hline
\end{tabular}

$$
m=\frac{B_{r} V_{m}}{\mu_{0}},
$$

where $B_{\mathrm{r}}$ is the residual magnetic flux of the permanent magnet and $V_{\mathrm{m}}$ is the volume of the magnet. ${ }^{13}$ Using the information in Table I and taking residual flux density for the neodymium magnet to be $1.15 \mathrm{~T} .{ }^{14}$ the magnetic dipole moment was estimated to be $2.21 \mathrm{~A}-\mathrm{m}^{2}$. Because the magnet's strength may differ from the manufacturer's specification, a second estimation of the dipole moment was obtained by varying the value of $m$ in the model until the energy curve predicted from the model matched the energy curve from the data. Using this approach, the dipole moment was found to be $2.1 \mathrm{~A}-\mathrm{m}^{2}$. The average error in the energy dissipated between the data and the model was about $8 \%$ for a dipole moment of $2.21 \mathrm{~A}-\mathrm{m}^{2}$ and about $2 \%$ for a dipole moment of $2.1 \mathrm{~A}-\mathrm{m}^{2}$. Unless otherwise specified, $2.1 \mathrm{~A}-\mathrm{m}^{2}$ will be used for the magnetic dipole moment. The accuracy of the model over the range of load resistances in Figure 4(b) indicates the electromechanical coupling factor expression is sufficiently accurate for describing the interaction between the magnet and the coil.

\subsection{Rotating test}

Having found the value of the dipole moment and verified the voltage induction model, the full model of the harvester can be examined. Figure 5 shows the rotating test, and Table II lists the parameters for the rotating test. The same coil and magnet are used in this test as in the free fall test. The system consists of a prototype energy harvester placed radially inside a bicycle wheel. An electric motor spins the wheel, and a laser tachometer measures the rotation rate. A wireless data acquisition (DAQ) system measures the output voltage across a $176 \Omega$ load resistor. This voltage is used to calculate the average power dissipated at a given rotation speed over a $60 \mathrm{~s}$ test.

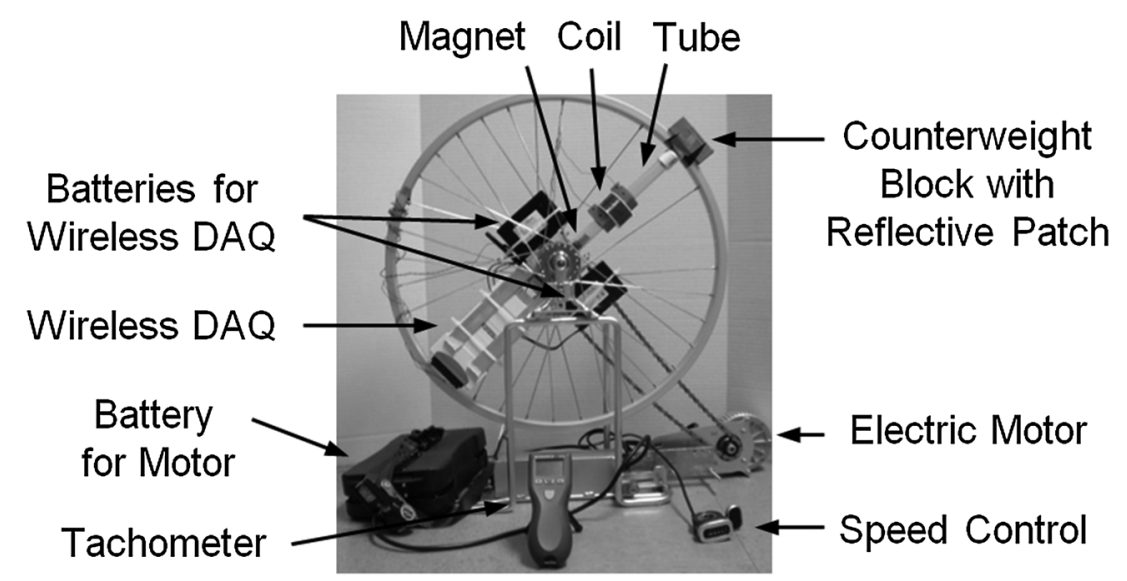

Figure 5. Rotating test apparatus. 
Table II. Parameters for the rotating test.

\begin{tabular}{|c|c|c|c|}
\hline & Parameter & Symbol & Value \\
\hline \multirow[t]{3}{*}{ Magnet } & Height & - & 0.75 in $(19.1 \mathrm{~mm})$ \\
\hline & Diameter & - & $0.5 \mathrm{in}(12.7 \mathrm{~mm})$ \\
\hline & Mass & M & $18 \mathrm{~g}$ \\
\hline \multirow[t]{7}{*}{ Coil } & Inner radius & $a_{1}$ & $0.509 \mathrm{in}(12.9 \mathrm{~mm})$ \\
\hline & Outer radius & $a_{2}$ & 1.02 in $(28.1 \mathrm{~mm})$ \\
\hline & Coil height & $h$ & 1.17 in $(29.7 \mathrm{~mm})$ \\
\hline & Wire diameter & $D_{w}$ & 0.0080 in $(0.203 \mathrm{~mm})$ \\
\hline & Coil resistance & $R_{\text {coil }}$ & $426 \Omega$ \\
\hline & Coil inductance & $L_{\text {coil }}$ & $0.998 \mathrm{H}$ \\
\hline & Distance from coil to center of rotation & $c_{1}$ & $4.63 \mathrm{in}(118 \mathrm{~mm})$ \\
\hline \multirow[t]{3}{*}{ Tube } & Radial position of the bottom of the tube & $r_{1}$ & 1.88 in $(47.6 \mathrm{~mm})$ \\
\hline & Radial position of the top of the tube & $r_{2}$ & 9.26 in $(235 \mathrm{~mm})$ \\
\hline & Tube length & 1 & 7.38 in $(187 \mathrm{~mm})$ \\
\hline \multirow[t]{2}{*}{ Other } & Load resistance & $R_{\text {load }}$ & $176 \Omega$ \\
\hline & DAQ sampling rate & - & $5000 \mathrm{~Hz}$ \\
\hline
\end{tabular}

Figure 6 shows the average load power to the load resistor versus rotation rate. The figure shows that increasing the rotation rate increases the power output until the average power reaches a maximum. Further increasing rotation speed causes the centrifugal effect on the magnet to become significant compared with the pull of gravity. The result is the magnet does not travel as far down the tube, less instantaneous velocity is achieved, and consequently, less power is produced. At sufficiently high speeds, gravity cannot overcome the centrifugal effects and the static friction from the tube. The magnet will cease to move, and no power will be produced.

A model without friction was first used to estimate the average load power, but this model underestimated the power output of the harvester at low speeds and failed to accurately predict the peak speed. Including Coulomb friction to the model allowed the model to more closely match the overall behavior of the data. By tilting the tube until the magnet began to move and then measuring the inclination angle, the static coefficient was estimated to be about 0.35 . Using this and setting the kinetic coefficient of friction to be 0.25 , the model predicts a maximum average power output of $3.5 \mathrm{~mW}$ at $45 \mathrm{rpm}$. The data shows a maximum power output of $3.3 \mathrm{~mW}$ at $44 \mathrm{rpm}$. The error between the model with friction and the data is about $3 \%$ over rotation rates below $45 \mathrm{rpm}$.

\section{APPLICATION IN FULL SCALE WIND TURBINES}

The power curve in Figure 6 will change once the harvester is placed inside a large scale wind turbine. Figure 7 shows simulation results for the average power output versus rotation rate for varying tube lengths and positions. The tube length is $l$, and the radial position of the bottom of the tube is $r_{1}$. The model uses the same parameters as the coil

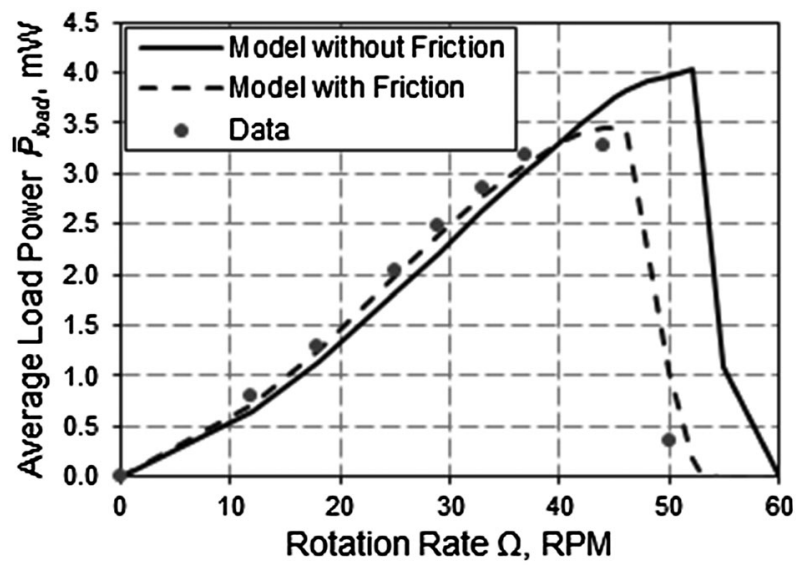

Figure 6. Average power versus rotation rate of the wheel. 


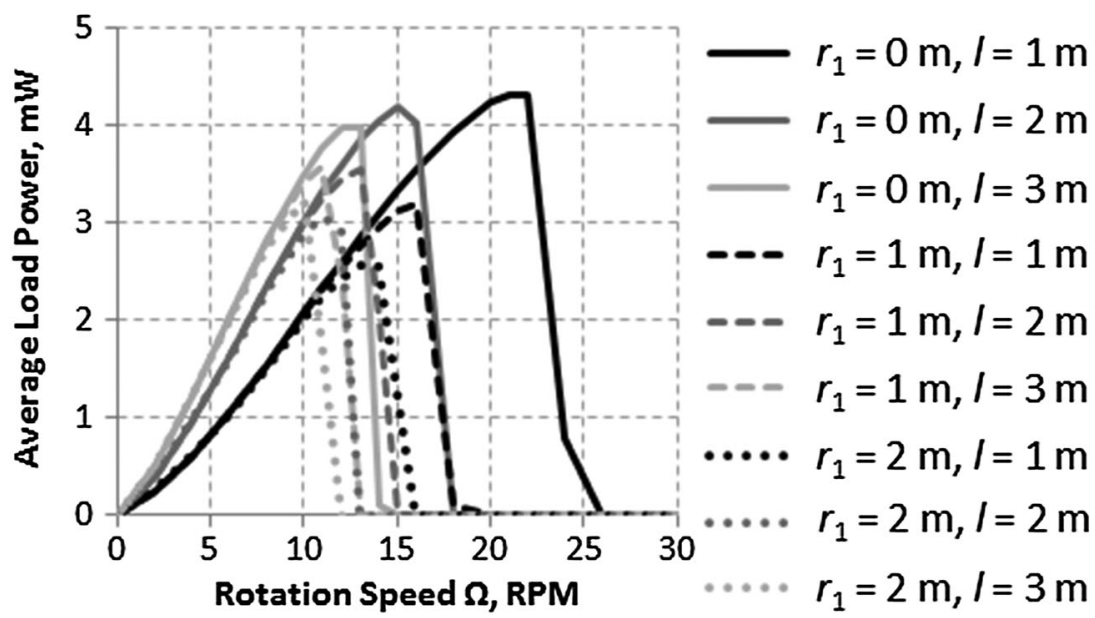

Figure 7. Average power versus rotation rate for various tube geometries.

and load resistor circuit from the experimental setup. The coil is placed in the middle of the tube for each simulation. Figure 7 shows that allowing the magnet to travel farther from the center of rotation (increasing either $r_{1}$ or $l$ ) decreases the range of rotation speeds for which the harvester produces power. For a given $r_{1}$ and rotation speed, increasing the tube length increases the power output until the tube length becomes long enough that the centrifugal effect on the magnet hinders its motion. The longer tube results in a longer travel distance for the magnet, which allows the magnet to reach higher velocities and generate more power. To maximize the power output of the harvester, the results in Figure 7 show that the device should be placed close to the root of the blade and the tube length should be as long as possible while still allowing the magnet to move over the range of operating speeds of the turbine. It should be noted that higher power outputs can be achieved by optimizing the coil geometry, optimizing the electrical properties of the circuit, using multiple coils per harvester, and using multiple harvesters per blade. ${ }^{12}$

A wireless SHM system can require between 100 and $500 \mathrm{~mW}$ of power to operate continuously. ${ }^{15,16}$ To help achieve the required power, a duty cycle system and an energy storage circuit could be employed to power the SHM equipment. Such a system would allow the harvester to store energy on a set of capacitors until the capacitors are sufficiently charged. Then the capacitors would power the SHM system for a few minutes per day. ${ }^{17}$ This approach reduces the instantaneous power demand on the harvester system and allows the harvester to power the SHM equipment.

\section{CONCLUSION}

This paper has outlined the development of an electromechanical energy harvester for use inside wind turbine blades. A model of the harvester was developed that incorporated the slider magnet's dynamics, the electrical circuit, and the coupling between the mechanics and the electrical circuit. A free fall test validated the electromechanical coupling model with the magnet having a magnetic dipole moment of $2.1 \mathrm{~A}-\mathrm{m}^{2}$. Next, a rotating test was used to examine the full system model. The prototype harvester was able to produce up to $3.3 \mathrm{~mW}$ at $44 \mathrm{rpm}$. The model with a static coefficient of friction of 0.35 and a kinetic coefficient of friction of 0.25 was able to match the data to within an error of about $3 \%$. Additional coils per harvester, additional harvesters per blade, and implementing a duty cycle system and an energy storage circuit would allow this harvester to power a SHM system inside a large scale wind turbine.

\section{ACKNOWLEDGEMENT}

This work was made possible, thanks to the support from the US Department of Commerce, National Institute of Standards and Technology, Technology Innovation Program, Cooperative Agreement Number 70NANB9H9007.

\section{REFERENCES}

1. Sutherland H. On the fatigue analysis of wind turbines. SAND99-0089, Sandia National Laboratories, 1999.

2. Simmermacher T, James GH, Hurtado JE. Structural health monitoring of wind turbines. Proceedings of the International Workshop on Structural Health Monitoring, Stanford, CA, 1997, 788-797. 
3. Pitchford CW. Impedance-based structural health monitoring of wind turbine blades. [Online]. Available: http://scholar. lib.vt.edu/theses/available/etd-09062007-140545/. (Accessed 12 January 2012).

4. AWEO.org. Size specifications of common industrial wind turbines. [Online]. Available: http://www.aweo.org/ windmodels.html. (Accessed 12 January 2012).

5. Jonkman J, Butterfield S, Musial W, Scott F. Definition of a 5-MW reference wind turbine for offshore system development. [Online]. Available: https://ceprofs.civil.tamu.edu/jzhang/ocen407/5MW\%20Reference\%20Turbine.pdf. (Accessed 12 January 2012).

6. Carlson C, Schlichting A, Ouellette S, Farinholt K, Park G. Energy Harvesting to Power Sensing Hardware Onboard Wind Turbine Blade. IMAC XXVIII, Jacksonville, FL, 2010.

7. Conrad SD. Development of an inertial generator for embedded applications in rotating environments. [Online]. Available: http://dspace.mit.edu/handle/1721.1/40947. (Accessed 12 January 2012).

8. Gu L, Livermore C. Passive self-turning energy harvester for extracting energy from rotational motion. Applied Physics Letters 2010; 97: 081904:1-081904:3. DOI: 10.1063/1.3481689.

9. Manla G, White NM, and Tudor J. Harvesting energy from vehicle wheels. 15th International Conference on Solid-state Sensors, Actuators and Microsystems. Transducers 2009; 1389-1392. DOI: 10.1109/SENSOR.2009.5285831.

10. Bedekar V, Oliver J, Priya S. Pen harvester for powering a pulse rate sensor. Journal of Physics D: Applied Physics 2009, 42: 105105:1-105105:9. DOI: 10.1088/0022-3727/42/10/105105.

11. Toh T, Mitcheson P, Holmes A, Yeatman E. A continuously rotating energy harvester with maximum power point tracking. Journal of Micromechanics and Microengineering 2008; 18: 104008:1-104008:7. DOI:10.1088/0960-1317/18/10/ 104008.

12. Joyce BS. Development of an electromagnetic energy harvester for monitoring wind turbine blades. [Online]. Available: http://scholar.lib.vt.edu/theses/available/etd-12202011-195538/. (Accessed 12 January 2012).

13. Sneller AJ, Mann BP. On the nonlinear electromagnetic coupling between a coil and an oscillating magnet. Journal of Physics D: Applied Physics 2010; 43: 295005:1-295005:10. DOI:10.1088/0022-3727/43/29/295005.

14. Magcraft. Permanent Magnet Selection and Design Handbook. National Imports LLC: Vienna, Virginia, $2007 ; 5$.

15. Physical Acoustics Corporation. 1282 acoustic emission wireless node \& system. [Online]. Available: http://www. mistrasgroup.com/products/company/publications/2\$Acoustic_Emission/1282_Wireless.pdf. (Accessed 12 January 2012).

16. Physical Acoustics Corporation. USB acoustic emission (AE) node. [Online]. Available: http://www.mistrasgroup. com/products/company/publications/2\$Acoustic_Emission/USB_AE_Node.pdf. (Accessed 12 January 2012).

17. Grisso BL, Kim J, Farmer JR, Ha DS, Inman DJ. Thermal Energy Harvested to Power Digital SHM Hardware. Virginia Space Grant Consortium, Norfolk, VA, 2008. 